\title{
A Hybrid Fault Tolerance System for Distributed Environment using Check Point Mechanism and Replication
}

\author{
S. Veerapandi \\ Research Scholar \\ School of information \\ Technology \\ Madurai Kamaraj University
}

\author{
S. Gavaskar, PhD \\ Assistant Professor \\ Department of Computer \\ Applications \\ Bharathiar University
}

\author{
A. Sumithra, PhD \\ Associate Professor \\ School of Computer Science \& \\ Engineering \\ VSB College of Technical \\ Campus
}

\begin{abstract}
Managing the distributed environment against the failures plays an important role nowadays. There are so many techniques evolved so far and each have their own merit and demerit. The efficiency of the algorithm depends on how much replication is done and upto what extent the fault tolerance has been achieved. We have here proposed a new method which uses both check point as well as the replication to ensure consistency in the distributed environment. Our method is also easy to implement.
\end{abstract}

\section{Keywords}

FTPA, PLR, GiFT

\section{INTRODUCTION}

A distributed system is a collection of independent computers that appears to its users as a single coherent system. Distributed Computing uses multiple geographically distant computers and solves big and complex task very efficiently. In other words, a distributed system is a collection of independent computers that appears to its users as a single coherent system. Computing power of idle hosts is utilized by distributed computing. Distributed systems offer a better price and performance than mainframes. Computing power can be added in small increments in distributed systems. In this way incremental growth can be achieved. Distributed systems allow many users access to a common computing resource thus provides resource sharing. Thus it allows many users to share expensive peripherals. It makes human-to-human communication easier. Examples of such distributed computing are online railway reservation system, air traffic control, internet banking etc. As the size of distributed system is increasing day by day chances of faults are increasing. Mean time to failure is decreasing with increase in size and complexity of distributed system. In large and dynamic distributed system millions of computing devices are working altogether and these millions of computing device are prone to failures. Failures of processors, disks, memory, power, and link failure are some examples of failures. Faults are inevitable in larger and dynamic distributed system. Faults may stop or halt execution of distributed system. It disturbs normal execution and may turn system execution in wrong direction.

In air traffic control, distributed disaster system, railways reservation system, internet banking a single fault may lead to huge loss of money and even human lives. In such a situation, inclusion of fault tolerance technique is essential. Fault Tolerance Techniques enable systems to perform tasks in the presence of faults [1]. There are high chances that more than one fault may occur in distributed system. For example more than one process may fail one by one or at a one time. Likewise more than one process may also fails in same manner. In such a situation simple fault a tolerance technique having capability to handle one fault are not suitable and does not solve the purpose. Such single fault tolerance algorithm fails to recover and restore the normal execution of dynamic distributed system in case of multiple faults. Handling more than one fault is a distinctive feature which is achieved using multiple fault tolerance technique. A multiple fault technique capable of tolerating $\mathrm{n}$ number of concurrent faults is known as kfaults tolerance technique. In some situation chain of faults occurs in such a way that the faults occurs when recovery of first is on progress and incomplete. Handling such types of multiple faults situation required a systematic approach and improved algorithms of multiple failure detection and recovery from multiple faults. Performance, scalability, robust, transparency, efficiency and consistency etc are some important issue with multiple fault tolerance implementation of distributed system.

In case of real time system multiple fault tolerance mechanism must provide performance in both the situation; fault free and faulty situation. Multiple node failures, process failure and failure of another node when recovery of failure of earlier node are some considered as a multiple faults occurrence in distributed system. To enhance the performance of multiple fault tolerance various overheads associate with every technique are required to minimize with improved algorithms. At the same time critical factor responsible for low performance need to be identified and ways need to explore to address these critical factors so that multiple fault capability can be improved with performance.

\section{VARIOUS FAULT TOLERANCE TECHNIQUES}

A. Replication Based Fault Tolerance Technique:

Replication based technique is one of the popular fault tolerance techniques [1]-[3]. A replica means multiple copies. Replication is a process of maintaining different copies of a data item or object. In replication techniques, request from client is forwarded to one of replica among a set of replicas. This technique is used for request that do not modify state of service. Replication adds redundancy in system. In this way failure of some nodes will not result in failure in system and thus faulttolerance is achieved as shown in fig 1. 


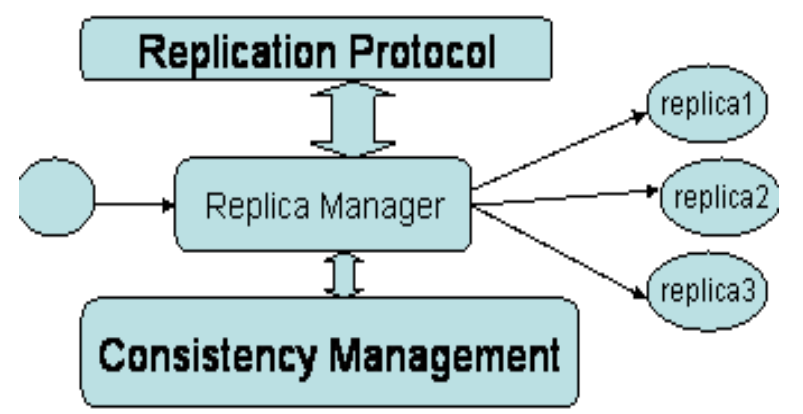

Fig1: Replication Based Technique

Replication protocol can be described using five generic phases. These phases are client contact, server coordination, execution, agreement, coordination and client response. Consistencies among replica, replica management, replica on demand, degree of replica etc. are some important issues in replication based fault tolerance technique. Major issues related to replication based techniques are consistency, degree of replica, replica on demand etc.

\subsection{Consistency}

Consistency among replicas is a major issue. Multiple copies of same entity causes problem of consistency due to update of any copy by one of the user. A replication protocol must ensure the consistency among all replicas of the same object. Consistency is ensured by some criterion. Many consistency criteria have been defined in the literature; linearizability [2], sequential consistency and causal consistency [3] etc. In all above cases, an operation is performed on the most recent state of the object. However consistency criteria differ in the definition of the most recent state. Primary-backup replication technique and active replication technique ensure consistency by linearizability. Both linearizability and sequential consistency define strong consistency criterion, whereas causal consistency defines a weak consistency criterion. Sequential consistency informally states that a multiprocessor program executes correctly if its result could have been produced by executing that program on single processor system. In order to have consistency an efficient strategy is required. Passive strategy and active strategy are main strategies. In a passive replication, only one primary execute requests and multicasts state changes to all replicas. This scheme avoids redundant computation of requests. It copes with non-deterministic service behavior. In active replica, client request is multicasts to all replicas. This means all replicas execute the request individually. In this way active replica takes less network resources than sending update. Active replica response to a fault is faster than passive. However, replica consistency usually requires deterministic replica behavior [4].Researcher proposed an algorithm that uses both active and passive strategies to implement optimistic replication protocol [5]. Researcher also proposed a simple protocol by combining the token with cache. This gives benefits of token as well as cache [6].There is still need of more simple, adaptive and practical replication protocol with adequate and sufficient ensured consistency

\subsection{Degree of Replica}

Number of replica is known as a degree of replication. In order to replicate an object a replication protocol is used. Primary-backup replication [27], voting [23], and primary-per partition protocol [24] are some of the replication protocol. A replication protocol must be practical and simple. The protocol must provide rigorously-proven yet simply-stated consistency guarantee with a reasonable performance. Niobe is such protocol purposed by researcher [25].Number of replicas must be sufficient. Large numbers of replicas will increase the cost of maintaining the consistency. Less number of replicas will affect the performance, scalability and multiple fault tolerance capability. Therefore, reasonable number replicas must be estimate as per system configuration and load. Researcher proposed adaptive replicas creation algorithm [26].There is further research scope to develop improved algorithm to maintain a rational replica number. Replica on demand is a feature that can be implemented to make more adaptive, flexible and dynamic. There is research scope to further improve protocols to achieve replication efficiently. There are some crucial requirements with replication protocol. These crucial requirements are support for a flexible number of replicas, strict consistency in the presence of network, disk, and machine failures and efficient common case read and write operations without requiring potentially expensive two or three-phase commit protocols.

\section{B. Process Level Redundancy}

This technique is mainly used as a fault tolerance for transient faults. A transient fault will eventually disappear without any apparent intervention. Transient faults are less severe but hard to diagnose and handle. It is caused by temporary malfunction of some system component. Some environmental interference also causes transient fault or faults. Transient faults are emerging as a critical concern in the reliability of distributed system. Hardware based fault tolerance is very costly hence software based fault tolerance is used to handle transient faults. Process-level redundancy (PLR) is a software based technique for transient fault tolerance, which leverages multiple cores for low overhead. PLR creates a set of redundant processes per application process as shown in fig 2 . It systematically compares the processes to guarantee correct execution. Redundancy at the process level allows the operating system to schedule freely the processes across all available hardware resources. PLR uses a software-centric approach to transient fault tolerance, which shifts the focus from ensuring correct hardware execution to ensuring correct software execution.

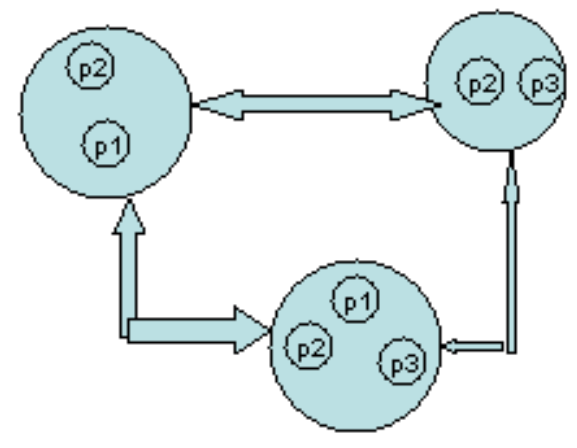

Fig 2:- N process Redundancy

As a result, many benign faults that do not propagate to affect program correctness can be safely ignored. PLR provides improved performance over existing software transient fault tolerance techniques with a 16.9 percent overhead for fault detection [7].However; PLR does not provide an adaptive and configurable fault tolerance on distributed systems. Further there is research scope to make PLR to support simultaneous faults by simply scaling the number of redundant processes and the majority vote logic. Future work remains in 
characterizing fault propagation, exploring methods for bounding the time in which faults remain undetected and performance improvement by minimizing the various overheads.

\subsection{Check pointing and Roll Back}

Checkpoint with rollback-recovery is a well-known technique. Checkpoint is an operation which stores the current state of computation in stable storage. Checkpoints are established during the normal execution of a program periodically. This information is saved on a stable storage so that it can be used in case of node failures. The information includes the process state, its environment, the value of registers, etc. When an error is detected, the process is roll backed to the last saved state [8]. Fig 3shown below gives an idea about this technique.

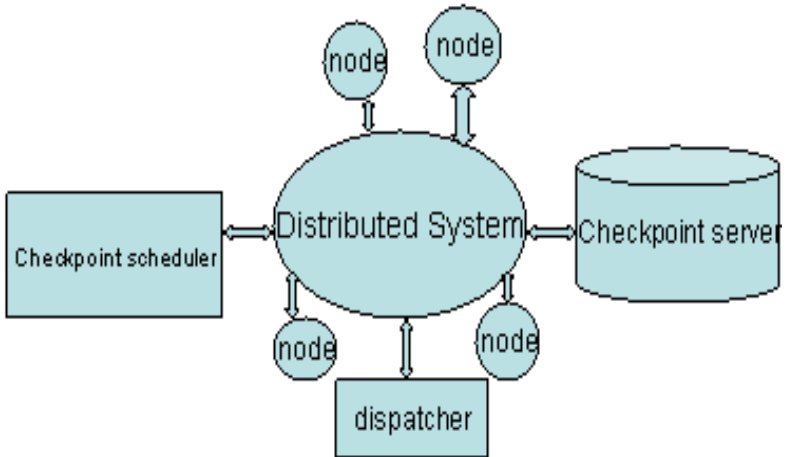

Fig3: Check pointing Technique

The main function of a recovery is to recover the system again in consistent and operation state as it continues to work in normal condition. Two most important types of rollback recovery are checkpoint based rollback recovery and log based rollback recovery. Checkpoint-based rollback recovery relies only on checkpoints .Log-based rollback-recovery combines' checkpointing with logging of non-deterministic events [9]. Coordinated checkpoint and uncoordinated checkpoint associated with message logging are the two main techniques used for saving the distributed execution state and recovering from system failures [10]. In coordinate check point processes coordinate their checkpoints in order to save a system wide consistent state. Coordinate check points are consistent set of checkpoints. These consistent check points are used to bound rollback propagation. Consistency is more in case of coordinate check points due to consistent set of checkpoints [9]. Coordinated checkpoint involves the rollback check point of all processes from the last snapshot when a faulty situation is detected, even when a single process crashes. Therefore recovery time is very large and it makes unsuitable for real time applications. In case of frequent failures and multiple faults coordinate check point technique cannot be used. Performance can be improved by decreasing the recovery time .Main reason for large recovery time is restarting all the initial state. Recovery time can be reduced by enabling the restart from last correct state instead of from very first state. There must be some mechanism to ensure restarting from last correct state will reach a state matching the the system, as before the crash.

Uncoordinated checkpoint protocols are designed to handle such critical issues to some extend. Message logging is combined with uncoordinated checkpoint to restart the system from last correct state.In Uncoordinated checkpoint protocols, all processes execute a checkpoint independently of the others so that recovery can be done independently with one another.
It is combined with message logging to ensure the complete description of a process execution state in case of its failure. Besides logging of all received messages, re-sending the same relevant messages in the same order to the crashed processes during their reexecution is also main function of message logging. There are three kinds of message logging protocols: optimistic, pessimistic and causal. Pessimistic protocols ensure that all messages received by a process are logged on reliable media before it sends information in the system. Log information on reliable media can be re-sent later and only if necessary during rollback.

Message logging optimistic protocols just ensure that all messages will eventually be logged. So, one usual way to implement optimistic logging is to log the messages on nonreliable media. Causal protocols log message information of a process in all causally dependent processes [11]. Check pointing based fault tolerance is very costly. Researcher proposed replication based check-pointing to improve the performance [12].There are many issues related to replication based check pointing fault-tolerance technique. These issues are mainly degree of replication, check pointing storage type and location, check pointing frequency, check point size and check point run time. At the same time researcher suggested an adaptive check pointing and replication to adapt dynamically the check pointing frequency and the number of replicas as a reaction on changing system properties (number of active resources, resource failure frequency and system load) [13]. In case of fault, the most important issue is efficient recovery in dynamic heterogeneous systems. Recovery under different numbers of processors is highly desirable. The fault tolerant and recover approaches must be suitable for applications with a need for adaptive or reactionary configuration control.

Researcher proposed flexible rollback recovery in dynamic heterogeneous computing for such crucial requirements [14]. Still overhead of this technique is significant and need to be address further. Performance of any fault tolerant technique depends on recovery time. Researchers and practitioners are trying to improve the recovery time by improving the recovery time. Conventional rollbackrecovery protocols redo the computation of the crashed process since the last checkpoint on a single processor. As a result, the recovery time of all protocols is no less than the time between the last checkpoint and the crash. Researcher proposed a new application-level faulttolerant approach for parallel applications called the Fault-Tolerant Parallel Algorithm (FTPA), which provides fast self-recovery. When fail-stop failures occur and are detected, all surviving processes recomputed the workload of failed processes in parallel. FTPA, however, requires the user to be involved in fault tolerance. In order to ease the FTPA implementation, Researcher developed Get it Fault-Tolerant (GiFT), a sourcetosource precompiled tool to automate the FTPA implementation. Researcher evaluates the performance of FTPA with parallel matrix multiplication and five kernels of NAS Parallel Benchmarks on a cluster system with 1,024 CPUs. The experimental results show that the performance of FTPA is better than the performance of the traditional check pointing approach due to fast recovery [15].However this is only suitable for large problem. If the problem size is not large enough, not all processes will contribute to parallel recomputing In order to tolerate multiple faults using checkpoint and recovery, three critical functionalities that are necessary for fault tolerance: a lightweight failure detection mechanism, dynamic process management that includes process migration, and a consistent checkpoint and recovery 
mechanism. Hugo Jung et al. proposed a technique to address this critical functionality [9].

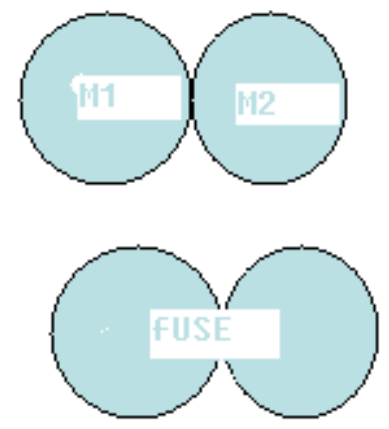

Fig 4: Fusion Process

\subsection{Fusion Based Technique}

Although replication method is widely used as a fault tolerance technique but number of backups is a main drawback. Number of backups increases drastically as coverage against number of faults increases. As the number of backup increases management of these backups is very costly. Fusion based techniques overcome this problem. It is emerging as a popular technique to handle multiple faults. Basically it is an alternate idea for fault tolerance that requires fewer backup machines than replication based approaches. In fusion based fault tolerance a technique, back up machines is used which is cross product of original computing machines. These backup machines are called as fusions corresponding to the given set of machines [28]. Overhead in fusion based techniques is very high during recovery from faults. Hence this technique is acceptable if probability of fault is low.

\section{PROPOSED TECHNIQUE}

Fault tolerance techniques enable systems to perform tasks in the presence of faults. Fault tolerance can be achieved through some kind of redundancy. The most common method used is checkpoint-restart [17]; an application is restarted from an earlier checkpoint or recovery point after a fault. This may result in the loss of some processing and applications may not be able to meet strict timing targets. Checkpointing is primarily used to avoid losing all the useful processing done before a fault has occurred [11]. Checkpointing consists of intermittently saving the state of a program in a reliable storage medium. Upon detection of a fault, previous consistent state is restored. In case of a fault, checkpointing enables the execution of a program to be resumed from a previous consistent state rather than resuming the execution from the beginning. In this way, the amount of useful processing lost because of the fault is significantly reduced.

\subsection{Types of checkpointing}

Depending on the programmer's intervention in process of checkpointing, it can be classified as follows:

\section{1) User triggered checkpointing}

These checkpointing schemes [18] require user interaction. These are generally employed where the user has the knowledge of the computation being performed and can decide the location of the checkpoints. The main problem is the identification of the checkpoint location by a user. This approach is well suited for long-running, computationintensive parallel applications, because of the minimal fault-free overhead. Indeed, there is no overhead during the normal execution of the application between the moments that the checkpoints are taken.

\section{2) Uncoordinated Checkpointing:}

In uncoordinated or independent checkpointing [16], processes do not coordinate their checkpointing activity and each process records its local checkpoint independently. In this way, each process becomes independent in deciding when to take checkpoint, i.e., each process may take a checkpoint when it is most convenient. It eliminates coordination overhead all together and forms a consistent global state on recovery after a fault. After a failure, a consistent global checkpoint is established by tracking the dependencies [11]. It may require cascaded rollbacks that may lead to the initial state due to domino-effect, i.e. the processes may resume from the beginning. It requires multiple checkpoints to be saved for each process and periodically invokes garbage collection algorithm to reclaim the checkpoints that are no longer needed. In this scheme, a process may take a useless checkpoint that will never be a part of global consistent state. Useless checkpoints incur overhead without advancing the recovery line.

\section{3) Coordinated Checkpointing}

In coordinated [16] or synchronous checkpointing, processes take checkpoints in such a manner that the resulting global state is consistent. Mostly it follows two-phase commit structure. In the first phase, processes take tentative checkpoints and in the second phase, these are made permanent. The main advantage is that only one permanent checkpoint and at most one tentative checkpoint is required to be stored. In case of a fault, processes rollback to last checkpointed state. A permanent checkpoint cannot be undone. It guarantees that the computation needed to reach the checkpointed state will not be repeated [11]. A tentative checkpoint, however, can be undone or changed to be a permanent checkpoint.

\section{4) Message Logging based checkpointing}

Messagelogging protocols [18] are popular for building systems that can tolerate process crash failures. Message logging and checkpointing can be used to provide fault tolerance in distributed systems in which all interprocess communication is through messages. Each message received by a process is saved in message log on stable storage. No coordination is required between the checkpointing of different processes or between message logging and checkpointing. When a process crashes, a new process is created in its place [11]. The new process [12] is given the appropriate recorded local state, and then the logged messages are replayed in the order the process originally received them. All message logging protocols require that once a crashed process recovers, its state needs to be consistent with the states of the other processes.

In computing, systems checkpoint is an essential for ensuring system availability. Checkpoint enables the system to continuously take snapshots of running applications; in the presence of a fault, the application can be rolled back to the most recent snapshot and continue execution with minimal downtime. Under error free execution, checkpoint incurs performance overhead. To make checkpointing attractive, the performance overhead must be minimized. All checkpoint mechanisms work on the basis of taking a snapshot of the running application. The snapshot required to recover an application consists of all application memory, opened files, sockets and IO devices. Our work also focuses on checkpointing applications but we concentrate on those that only require memory state recovery. The application memory state has the largest footprint and therefore will be the most time--- consuming. 
In this work the I/O traffic is buffered during each checkpoint and if a recovery is required the $\mathrm{I} / \mathrm{O}$ is played back so that the application receives the same input during the second execution. The biggest factor to affect the performance overhead is the method adopted for the memory duplication process. The most rudimentary approach is to perform a full memory copy of the application at each checkpoint interval. This strategy, however, causes a great performance overhead since it requires a large amount of memory bandwidth. For this reason, the most widely accepted approach is to duplicate only a select region of application memory during each checkpoint. This approach is called incremental checkpoint [19]-[23]. The method of selecting which data to save and at what time interval varies between the different checkpoint types. In this we are implementing a memory space reduction scheme by deleting the unwanted checkpoints which are not at all further required. By this way our scheme proves to be space saving as well as time complexity is also reduced. Even if we need the deleted checkpoints detail that can also be got by a replication scheme. The full memory copy of the application at each checkpoint interval is copied into a secondary storage such as the hard disk of the system.

\section{CONCLUSION}

In this paper we have proposed an improved and efficient technique which ensures the consistency in the distributed environment using java RMI. The proposed technique involves lock leased protocol for performing the various write-write or read-write operation. Simultaneous concurrent read operations are possible in this environment. Our method is very simple and easy to be implemented. It efficiently reduces the checkpointing overhead by saving the checkpoints on local hard disk as well as only selected data in memory.

\section{REFERENCES}

[1] M. Wiesmann, F. Pedone, A. Schiper, B. Kemme, G. Alonso," Understanding Replication in Databases and Distributed Systems," Research supported by EPFLETHZ DRAGON project and OFES).

[2] M. Herlihy and J. Wing. "Linearizability: a correctness condition for concurrent objects," ACM Trans. on Progr. Languages and Syst., 12(3):463-492, 1990. (IJIDCS) International Journal on Internet and Distributed Computing Systems. Vol: 1 No: 1, 39

[3] M. Ahamad, P.W. Hutto, G. Neiger, J.E. Burns, and P. Kohli., "Causal Memory:Definitions, implementations and Programming," TR GIT-CC-93/55, Georgia Institute of Technology, July 94.

[4] H.P. Reiser, M.J. Danel, and F.J. Hauck., “ A flexible replication framework for scalable andreliable .net services.," In Proc. of the IADIS Int. Conf. on Applied Computing, volume1, pages 161-169, 2005.

[5] A. Kale, U. Bharambe, "Highly available fault tolerant distributed computing using reflection and replication," Proceedings of the International Conference on Advances in Computing, Communication and Control ,Mumbai, India Pages: 251-256, , 2009

[6] X. China, "Token-Based Sequential Consistency in Asynchronous Distributed System ," 17 th Internaional Conference on Advanced Information Networking and Applications (AINA'03),March 27-29, ISBN: 0-76951906-7

[7] A. Shye, , J. Blomstedt, , T. Moseley,V. Reddi, , and Daniel A. Connors, "PLR: A Software Approach to
Transient Fault Tolerance for Multicore Architectures" Pp135-148.

[8] V. Agarwal, Fault Tolerance in Distributed Systems, I. Institute of Technology Kanpur, www.cse.iitk.ac.in/report-repository, 2004. ,

[9] H. Jung, D. Shin, H. Kim, and Heon Y. Lee, "Design and Implementation of Multiple FaultTolerant MPI over Myrinet (M3) ," SC|05 Nov 1218,2005, Seattle, Washington, USA Copyright 2005 ACM.

[10] M. Elnozahy, L. Alvisi, Y. M. Wang, and D. B. Johnson. A survey of rollback-recovery protocols in message passing systems. Technical Report CMU-CS-96-81, School of Computer Science, Carnegie Mellon University, Pittsburgh, PA, USA, October 1996.

[11] L. Alvisi and K. Marzullo. Message logging : Pessimistic, optimistic, and causal. In Proceedings of the $15^{\text {th }}$ International Conference on Distributed Computing,Systems (ICDCS 1995), pages ,229-236. IEEE CS Press, May-June 1995.

[12] J. Walters and V. Chaudhary," Replication-Based Fault Tolerance for MPI Applications," Ieee Transactions On Parallel And Distributed Systems, Vol. 20, No. 7, July 2009

[13] M Chtepen, F.. Claeys, B. Dhoedt, , and P. Vanrolleghem," Adaptive Task Checkpointing and Replication:Toward Efficient Fault-Tolerant Grids", IEE Transactions on Parallel and Distributed Systems, Vol. 20, No. 2, Feb 2009

[14] S. Jafar, A. Krings, and T. Gautier," Flexible Rollback Recovery in Dynamic Heterogeneous Grid Computing", IEEE Transactions On Dependable and Secure Computing, Vol. 6, No. 1, Jan-Mar 2009

[15] X. Yang, Y. Du, Panfeng W. Fu, and Jia "FTPA: Supporting Fault-Tolerant Parallel Computing through Parallel Recomputing," Ieee Transactions On Parallel And Distributed Systems, Vol. 20, No. 10, October 2009

[16] S. Gorender, and M Raynal, "An Adaptive Programming Model for Fault-Tolerant Distributed Computing" Ieee Transactions On Dependable And Secure Computing, Vol. 4, No. 1, January-March 2007.

[17] A. Luckow B. Schnor, „“Adaptive Checkpoint Replication for Supporting the Fault Tolerance of Applications in the Grid,“ Seventh IEEE International Symposium on Network Computing and Applications, 2008 IEEE.

[18] A. Bouteiller, F. Cappello, T. H Krawezik, Pi Lemarinier, F Magniette, "MPICH-V2: a Fault Tolerant MPI for Volatile Nodes based on Pessimistic Sender Based Message Logging, " SC'03, NoV 15-21, 2003, Phoenix, Arizona, USA Copyright 2003 ACM 1-58113-695$1 / 03 / 001$

[19] I. Saha, D. Mukhopadhyay and S. Banerjee, "Designing Reliable Architecture For Stateful Fault Tolerance," Proceedings of the Seventh International Conference on Parallel and Distributed Computing,Applications and Technologies (PDCAT'06) 2006 .

[20] N. Gorde, S. Aggarwal, “A Fault Tolerance Scheme for Hierarchical Dynamic Schedulers in Grid” International 
Conference on Parallel Processing Workshops, 2008 IEEE

[21] Y. Li, , Z. Lan, , P. Gujrati and , X. Sun, , "FaultAwareRuntime Strategies for High-Performance Computing," IEEE Transactions on Parallel And Distributed Systems, Vol. 20, No. 4, April 2009

[22] G. Jakadeesan, D. Goswami, "A Classification-Based Approach to Fault-Tolerance Support in Parallel Programs", International Conference on Parallel and Distributed Computing, Applications and Technologies, 2009 IEEE.

[23] D.K. Gifford, "Weighted voting for replicated data," In SOSP '79: Proc. of the seventh ACM symposium on Operating systems principles, pages 150-162, 1979.

[24] J. Osrael, L. Froihofer, K.M. Goeschka, S. Beyer,P. Gald'amez, , and F. Mũnoz. "A system architecture for enhanced availability of tightly coupled distributed systems," In Proc. of 1st Int. Conf. on Availability, Reliability, and Security.IEEE, 2006

[25] J Maccormick1, C Thekkath, M.Jager,K. Roomp, and L. Peterson , "Niobe: A Practical Replication Protocol." ACM Journal Name, Vol. V, No. N, Month 20YY.
[26] Cao Huaihu, Zhu Jianming, "An Adaptive Replicas Creation Algorithm with Fault Tolerance in the Distributed Storage Network" 2008 IEEE.

[27] N. Budhiraja, K. Marzullo, F.B. Schneider, and S. Toueg. The Primary-Backup Approach. In Sape Mullender, editor, Distributed Systems, pages 199-216. ACM Press, 1993.

[28] V.K Garg,. "Implementing fault-tolerant services using fused state machines," Tech-nical Report ECE-PDS2010- 001, Parallel and Distributed Systems Laboratory,ECE Dept. University of Texas at Austin (2010).

[29] N. Xiong, M. Cao, J. He and L. Shu, "A Survey on Faulttolerance in Distributed Network Systems," 2009 International Conference on Computational Science, 978- 0-7695-3823-5/09

[30] D. Tian , K. Wu X. Li, "A Novel Adaptive Failure Detector for Distributed Systems," Proceedings of the 2008 International Conference on Networking, Architecture, and Storage (C2008, ISBN: 978-0-76953187-8 